\title{
Repair of internal carotid artery injury with aneurysm clip during endoscopic endonasal surgery: illustrative case
}

\author{
David Fustero de Miguel, MD, Laura Beatriz López López, MD, Amanda Avedillo Ruidíaz, MD, Javier Orduna Martínez, MD, \\ Juan Casado Pellejero, MD, and Jesús Adrián Moles Herbera, MD
}

Department of Neurosurgery, University Hospital Miguel Servet, Zaragoza, Spain

\begin{abstract}
BACKGROUND One of the most feared and dangerous scenarios that can appear during an endoscopic endonasal surgery (EES) is the iatrogenic injury of the internal carotid artery (ICA). Several methods, along with a variety of outcomes, have been described to deal with this complication. To the authors' knowledge, this is the first report on the use of a Yasargil-type aneurysm clip to solve an ICA injury, preserving the artery's patency and having a long-term follow-up. The authors discuss the advantages and disadvantages of other vessel preservation techniques compared with clipping.

OBSERVATIONS A visually impaired 56-year-old woman was diagnosed with a giant nonfunctional pituitary tumor that invaded the sphenoidal sinus, anterior and posterior ethmoidal cells, and both cavernous sinuses, with suprasellar extension and optochiasmatic compression. The patient underwent EES, and during the final resection phase her left ICA was injured, with massive hemorrhage.
\end{abstract}

LESSONS ICA injury during endoscopic skull base surgery carries high mortality and morbidity; it is essential to maintain carotid flow when possible to avoid short-term and long-term consequences. There are several techniques depicted in the literature to deal with this situation. The authors report the use of a Yasargil mini-clip to deal with the injury for a positive outcome: primary hemostasis, vessel preservation, and no postoperative complications.

https://thejns.org/doi/abs/10.3171/CASE2098

KEYWORDS complications; endoscopic endonasal surgery; internal carotid artery injury; skull base surgery; surgical clip; pituitary adenoma

Internal carotid artery (ICA) injury during endoscopic endonasal surgery (EES) for approaching of skull base lesions is one of the most feared and severe complications that a skull base surgeon may tackle. Its incidence in functional endoscopic sinus surgery is extremely rare, with 28 cases reported in the review by Valentine and Wormald ${ }^{1}$ and a frequency of $0.025 \%$ noted in the longest published series. ${ }^{2}$ This incidence increases to $0.3 \%,{ }^{3} 0.7 \%,{ }^{4}$ or $0.9 \%{ }^{5}$ in transsellar endoscopic surgery, specifically to $0.9 \%$ in expanded approaches, and in the chondroid subgroup tumors it can even rise to $2 \% .^{3}$ In a report of microsurgical transsphenoidal surgery that reviews the most cases, the frequency of this complication is similar: $0.9 \%{ }^{7}$ There are issues when evaluating these incidences. First, there are series that mix microsurgical approaches with endoscopic ones as well as lesions with a low risk of vascular injury with those of high risk. Second, due to unpublished cases, the possibility of underestimation should not be ignored.

Technically, aside from the inherent difficulties due to the narrow corridor during the approach, the view is compromised by bleeding, which hinders its resolution. Above all, a massive hemorrhage occurs that can lead to the patient's exsanguination in a few minutes. Consequently, this event must be solved immediately, first by trying to save the patient's life and second by avoiding long-term catastrophic consequences in neurological function.

A variety of techniques have been described in the literature to deal with this complication; however, among them, vascular injury reconstruction using an aneurysm clip has been scarcely used given its technical complexity. We describe the first published case of endoscopic repair of an ICA injury using a Yasargil mini-clip (Aesculap) with ulterior preservation of vascular flow without long-term complications.

\section{Illustrative Case}

A 56-year-old woman was diagnosed in April 2016 with a nonfunctioning invasive macroadenoma, the first symptom of which was a partially recovered left amaurosis. After preoperative studies that included magnetic resonance imaging (MRI), angio-MRI, and a skull

ABBREVIATIONS BTO = balloon test occlusion; $\mathrm{CT}=$ computed tomography; $\mathrm{EES}=$ endoscopic endonasal surgery; $\mathrm{ICA}=$ internal carotid artery; $\mathrm{MRI}=$ magnetic resonance imaging; $P A=$ pseudoaneurysm.

INCLUDE WHEN CITING Published February 8, 2021; DOI: 10.3171/CASE2098.

SUBMITTED November 10, 2020. ACCEPTED November 18, 2020.

(C) 2021 The authors, CC BY-NC-ND 4.0 (http://creativecommons.org/licenses/by-nc-nd/4.0/), 
base computed tomography (CT) scan for neuronavigation (StealthStation, Medtronic), a giant sellar and parasellar lesion was diagnosed, invading the sphenoidal and ethmoidal bone anatomy, with impairment of both cavernous sinuses, left ICA encasement, and chiasmatic and left optic nerve compression (Fig. 1).

Subsequently, informed consent was obtained from the patient, and an endoscopic endonasal transsphenoidal approach was performed in collaboration with the Ear, Nose, and Throat Department. After the approach and partial dissection of the lesion, during the final resection phase, the cavernous portion of the left ICA was injured with a Frazier suction tube, probably as a result of an avulsion of a dependent branch (presumably the meningohypophyseal trunk in its exit from the ICA). The bleeding was controlled by means of the application of a 3-mm Yasargil mini-clip (Fig. 2), confirming the intraoperative preservation of adequate flow through the injured vessel with a Doppler probe (Mizuho Surgical). The surgery was finished, and the skull base was reconstructed in a multilayer fashion, covering the clip with an autologous fat graft. Then a Duragen dural graft (Integra) was applied and a nasoseptal flap was used posteriorly to cover the defect. ${ }^{8}$ Afterwards, Duraseal glue (Integra) sealed the reconstruction. ${ }^{9}$ This multilayer technique is paramount in preventing an erosion in the closure provoked by the protruding clip.

The immediate angiographic control revealed patency of the ICA with no complications and minimal stenosis (Fig. 3). The patient did not show any neurological deficits derived from the event (Video 1). Afterwards, the remaining lesion in the left cavernous sinus was treated using Gamma Knife radiosurgery, without any unwanted incidents. During the 4-year follow-up period, the patient recovered normal visual fields and did not require hormone replacement therapy. The last angiography performed 4 years after the surgery did not show any complications.

VIDEO 1. Clip including preoperative studies, intraoperative images showing ICA injury and its management, and postoperative results. Click here to view.

\section{Discussion}

In the last three decades, endoscopic skull base surgery has had important developments regarding the extension of its indications and types of approach.

There is wide agreement about conducting a thorough presurgical study that includes a cranial thin-slice CT scan, brain MRI, and angio$\mathrm{CT}$ in order to comprehend the characteristics of the tumoral pathology and the surrounding vascular anatomy. Although performing it on a regular basis is not recommended in all patients undergoing EES, ${ }^{3}$ angiography should be considered specifically if its associated with a balloon test occlusion (BTO) in those lesions that entail a high risk of vascular injury..$^{10,11}$ Therefore, if the BTO is positive and we are dealing with a carotid laceration, we may sacrifice the vessel, if necessary, after being confident that we can avoid ischemic stroke and neurological sequelae. However, with a negative BTO, we could either reconsider the surgical goal and aggressiveness or perform a prophylactic bypass. Nevertheless, despite a positive BTO, Lippert et al. reported a $10 \%$ incidence of strokes after ICA sacrifice. ${ }^{12}$

Several factors that imply a high risk of ICA injury have been observed. Among them, tumor-like chordomas or chondrosarcomas; adherence, encasement, or invasion of the ICA by the lesion; the need for ICA manipulation for tumoral resection; expanded approaches in the coronal plane; postradiotherapy; previous surgery; acromegaly; a history of bromocriptine therapy; and so on are more well known. ${ }^{1,13-15}$

Various studies in cadaveric specimens or using radiological images highlight the importance of a previous exhaustive evaluation of the region's anatomy in order to prevent iatrogenic vascular lesions. The ICA protrudes into the sphenoid sinus in approximately $71 \%$ of cases, ${ }^{16}$ and the typical distance between ICAs in a normal-sized sella is around $17 \mathrm{~mm} .{ }^{17}$ However, this distance can be smaller, with carotid arteries located as close as $4 \mathrm{~mm}$ from the midline within the sella in $10 \%$ of cases. ${ }^{16}$ Fujii et al. demonstrated that the bony wall that protects the ICA in the sphenoid sinus is less than $0.5 \mathrm{~mm}$ thick in $88 \%$ of cases, and areas with the absence of bone between the artery and the sinus were present in $8 \%$ of cases, thus increasing the risk of unintentional injury. ${ }^{17}$ Additionally, in $16.3 \%$ of the cases, the intrasphenoidal boney septum ends in the ICA canal, with the inherent possibility of injuring the ICA while removing it. ${ }^{18}$

An ICA lesion triggers high-flow bleeding that, along with the narrow space available in EES approaches, reduces visibility in the surgical field, hence hampering direct control of the hemorrhage. Once the bleeding spot is identified and the hemorrhage is managed by packing, it has to be decided whether to preserve the vessel or to occlude and sacrifice the artery instead; these goals can be achieved with direct or indirect surgical techniques or endovascular treatment. The right decision depends on the injury type and location, the surgical field range, and adequate collateral circulation. Hereunder, some of the available techniques reported in the literature to manage this complication are described.
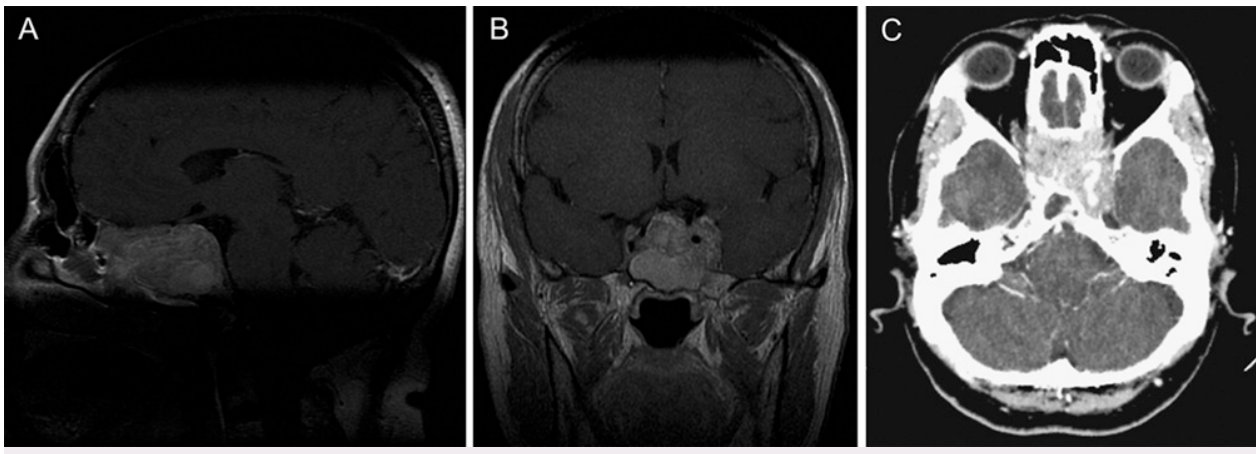

FIG. 1. Preoperative sagittal (A) and coronal (B) T1-weighted gadolinium-enhanced MRI showing invasive sellar and parasellar mass, affecting both cavernous sinuses, with encasement of left ICA. C: Preoperative CT angiography showing encroachment of left ICA. 

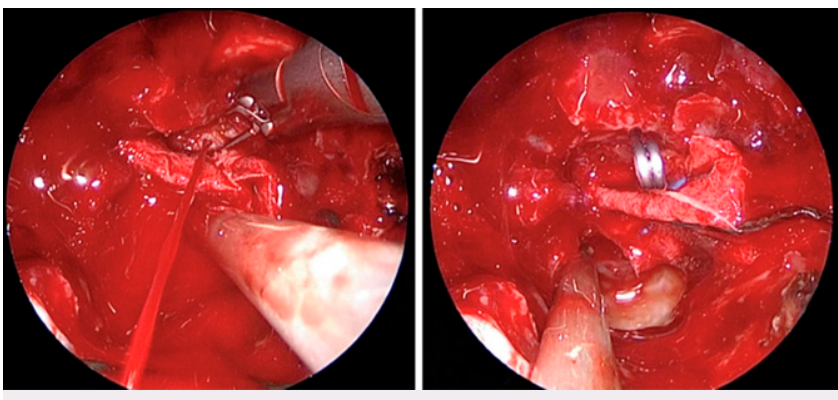

FIG. 2. Left: Intraoperative endoscopic view showing a jet of blood from the injured left ICA. Right: Intraoperative endoscopic view after successful placement of an aneurysm clip, controlling the hemorrhage.

Direct suture has been described as a successful treatment in 3 articles, which include microsurgical and endoscopic procedures. Two of the articles have little to no details of any further result or procedure, ${ }^{15,19}$ whereas Cobb et al. report a direct suture of the laceration with Prolene 9.0 after controlling the bleeding with an endovascular balloon proximal to the injury. ${ }^{20}$

Because of the reduced and narrow surgical field, placement of an aneurysm clip using an endoscopic approach is a risky and challenging maneuver. Nevertheless, this is a feasible way of preserving ICA permeability, as proven in our case. Even though this procedure may cause some grade of vessel stenosis, no neurological sequelae have been noticed. Carotid clipping can also be used to occlude the ICA safely. $3,21,22$ Furthermore, studies in artificial or animal models have proven that this is a technique that achieves quick hemostasis, preserves the patency of the damaged artery, minimizes blood loss, and has no evidence of postsurgical bleeding or pseudoaneurysms (PAs). ${ }^{23}$

Variants to the traditional aneurysm clip have been described in the literature, such as the Sundt-type clip graft, which consists of a circumferential device that involves the damaged vessel inside. However, it can cause vessel stenosis leading to its occlusion, as in the cases reported by Gardner et al. ${ }^{3}$ and others. ${ }^{15,19}$

To our knowledge, this is the first report that describes the use of a Yasargil-type aneurysm clip to solve an ICA injury, preserving its patency, except for the case of a petroclival meningioma published by Gardner et al. in $2016 .{ }^{13}$ In that paper, some images of the procedure
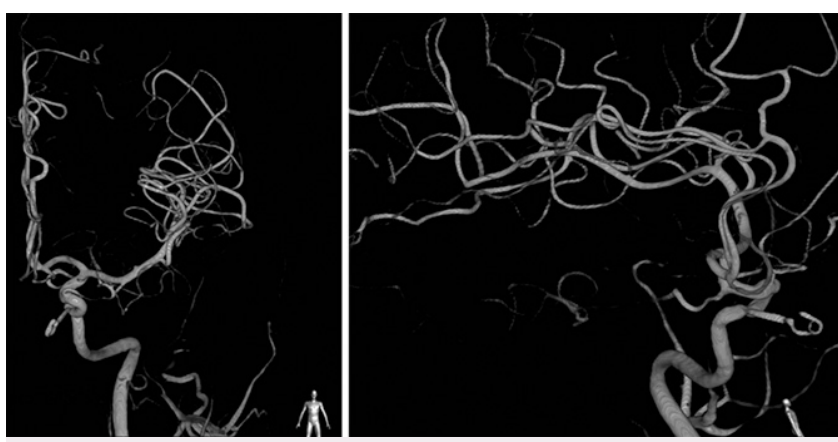

FIG. 3. Postoperative anteroposterior (left) and lateral (right) threedimensional angiography showing no evidence of a PA and verifying vessel patency. are shown without details regarding the technique, the long-term followup, or the patient functional status.

The use of extended endoscopic endonasal approaches for microsurgical clipping of intracranial aneurysms has been reported previously. ${ }^{24,25}$ Nevertheless, this technique is reserved for wellselected patients under the care of highly experienced teams. ${ }^{9} \mathrm{Re}$ gardless, this article shows the possibility of treating an acute iatrogenic injury of the ICA with an aneurysm clip, and it is beyond the scope of this article to discuss endoscopic endonasal intracranial aneurysm clipping.

Bipolar coagulation might be a good option for small injuries $(<3 \mathrm{~mm})$, considering that it could be associated with carotid stenosis. Its use for treating the defect and preserving carotid patency, as well as for complete occlusion of the artery, has been described. In an experimental study with animal specimens, bipolar coagulation provided better hemostasis than the muscular graft, although it was worse than the clip, with a higher risk of rebleeding and PA formation than with the clip. ${ }^{23}$ As reported in the literature, it is common to combine this procedure with the posterior placement of a muscle graft, with around $50 \%$ preservation of carotid flow. $3,22,26$

The muscle graft is obtained from the sternocleidomastoid, temporal, rectus abdominis, quadriceps, or longus capitis muscle endoscopically. ${ }^{26}$ It is the most achievable alternative if direct closure of the defect on the vessel wall is not possible. Experimental model studies show good primary hemostasis with a higher risk of PA formation. 1,23,27 Multiple articles describe the use of muscle graft alone or combined with other techniques to control bleeding. 2,22,26,28-30

Undoubtedly, it is interesting to practice the different techniques described above in artificial, animal, or cadaveric models. This increases surgical experience and confidence in surgeons, improving their skills and decision-making capability when a complication of this magnitude takes place..$^{23,27,31-33}$

In the literature, the most commonly described strategy to achieve initial hemostasis is to perform a tamponade or packing and/or placement of a muscle graft, followed by angiography to complete an initial diagnosis and possible treatment. ${ }^{13}$ Consequently, it is recommended to have a neurointerventional radiology service available in those centers where EES is performed. ${ }^{34}$

Over the last years, endovascular treatment has increased its role because of the release of new devices and innovation in techniques of its implementation. ${ }^{34}$ Formerly, predominant endovascular treatment was limited to the sacrifice of the ICA. ${ }^{35}$ Nowadays, however, the aim of these techniques is to achieve lesion control and preserve the vessel. ${ }^{36}$ As we have previously described, even in those patients who have a favorable occlusion test, carotid arteries are at risk of causing an ischemic stroke, ${ }^{37}$ and the available information about the long-term consequences of carotid occlusion is scarce. ${ }^{3}$

The endovascular preservation techniques harbor two concerning drawbacks. The first one is the need to introduce periprocedural anticoagulant prophylaxis, subsequently replaced by dual antiplatelet therapy, if a covered stent or flow diverter is deployed, with the concomitant hemorrhagic risk. Nevertheless, different series show that previous surgery should not be an absolute contraindication for this therapy. ${ }^{34,36}$

The second hindrance for covered stents is the limited longitudinal flexibility of these devices that makes their placement in a tortuous ICA sometimes challenging. ${ }^{15}$ To solve this problem, different techniques have been developed such as reconstructive endovascular treatment with overlapping common stents or stent-assisted coiling, among other things. 38,39 Their long-term outcomes should be carefully evaluated. 
Among the ICA injury complications that we may find (cavernous sinus thrombosis, embolisms, caroticocavernous fistula, cerebral ischemia, vasospasm), the appearance of a PA is the most frequent, regardless of the use of reconstructive or occlusive techniques. ${ }^{40}$ Some papers claim that the risk of its occurrence is higher when using indirect techniques, such as packing or a muscle patch. ${ }^{15,19}$ The review by Valentine and Wormald highlights a $60 \%$ incidence of PA formation after intraoperative ICA rupture events using local packing treatment. ${ }^{1}$ For its management, most authors prefer endovascular techniques, with covered stents or flow diversion devices being the treatments of choice. ${ }^{34}$

Although the presented case had an adequate evolution without sequelae, carotid laceration is a complication with a poor prognosis. Registered mortality is $15 \%$ and permanent morbidity is $26 \%,{ }^{1}$ and these rates could be underestimated due to publication bias or the short length of the follow-up because the mentioned complications such as PA may appear years after an ICA lesion. ${ }^{14}$ Therefore, a long follow-up with angio-CT, angio-MRI, or angiography is recommended after 1 month, 6 months, and several years from the event with the aim of identifying and treating the occurrence of late complications. $3,13,14,35$

\section{Observations}

The use of an aneurysm clip to deal with an iatrogenic ICA lesion during EES in selected cases is feasible and may preserve vessel flow with long-term security. To our knowledge, this is the first report that describes the use of a Yasargil-type aneurysm clip to solve an ICA injury, preserving its patency, with a long-term follow-up and information about patient functional status. Gardner et al., in 2016,13 described its use in a petroclival meningioma, without going into details regarding the technique or follow-up.

\section{Lessons}

latrogenic injury of the ICA during EES involves a high mortality and morbidity rate in the short and long terms. Hence, it is paramount to avoid it through an individualized anatomical study, preoperative measures such as BTO, and intraoperative ones that include neuronavigation and the use of a Doppler probe. In this case, we describe one of the technical possibilities to solve it, which did not involve either ICA sacrifice or any associated morbidity. The obvious limitations of this study are the scarcity of experiences with this technique published in the literature, with just two cases (including this one) reported, and its applicability in selected types of ICA injuries.

\section{References}

1. Valentine R, Wormald PJ. Carotid artery injury after endonasal surgery. Otolaryngol Clin North Am. 2011;44(5):1059-1079.

2. Weidenbecher $M$, Huk WJ, Iro $H$. Internal carotid artery injury during functional endoscopic sinus surgery and its management. Eur Arch Otorhinolaryngol. 2005;262(8):640-645.

3. Gardner PA, Tormenti MJ, Pant $\mathrm{H}$, et al. Carotid artery injury during endoscopic endonasal skull base surgery: incidence and outcomes. Neurosurgery. 2013;73(2)(suppl operative):ons261-ons270.

4. Cappabianca P, Cavallo LM, Colao A, et al. Surgical complications associated with the endoscopic endonasal transsphenoidal approach for pituitary adenomas. J Neurosurg. 2002;97(2):293-298.

5. Gondim JA, Almeida JPC, Albuquerque LAF, et al. Endoscopic endonasal approach for pituitary adenoma: surgical complications in 301 patients. Pituitary. 2011;14(2):174-183.

6. Frank G, Sciarretta V, Calbucci F, et al. The endoscopic transnasal transsphenoidal approach for the treatment of cranial base chordomas and chondrosarcomas. Neurosurgery. 2006; 59(suppl 1):ONS50-ONS57.
7. Raymond J, Hardy J, Czepko R, et al. Arterial injuries in transsphenoidal surgery for pituitary adenoma; the role of angiography and endovascular treatment. AJNR Am J Neuroradiol. 1997; 18(4):655-665.

8. Hadad G, Bassagasteguy L, Carrau RL, et al. A novel reconstructive technique after endoscopic expanded endonasal approaches: vascular pedicle nasoseptal flap. Laryngoscope. 2006;116(10):1882-1886.

9. Gardner PA, Vaz-Guimaraes F, Jankowitz B, et al. Endoscopic endonasal clipping of intracranial aneurysms: surgical technique and results. World Neurosurg. 2015;84(5):1380-1393.

10. AIQahtani A, Castelnuovo P, Nicolai $P$, et al. Injury of the internal carotid artery during endoscopic skull base surgery: prevention and management protocol. Otolaryngol Clin North Am. 2016;49(1): 237-252.

11. AIQahtani A, London NR Jr, Castelnuovo P, et al. Assessment of factors associated with internal carotid injury in expanded endoscopic endonasal skull base surgery. JAMA Otolaryngol Head Neck Surg. 2020;146(4):364-372.

12. Lippert BM, Ringel $K$, Stoeter $P$, et al. Stentgraft-implantation for treatment of internal carotid artery injury during endonasal sinus surgery. Am J Rhinol. 2007;21(4):520-524.

13. Gardner PA, Snyderman CH, Fernandez-Miranda JC, et al. Management of major vascular injury during endoscopic endonasal skull base surgery. Otolaryngol Clin North Am. 2016;49(3): 819-828.

14. Solares CA, Ong YK, Carrau RL, et al. Prevention and management of vascular injuries in endoscopic surgery of the sinonasal tract and skull base. Otolaryngol Clin North Am. 2010;43(4): 817-825.

15. Oskouian RJ, Kelly DF, Laws ERJ Jr. Vascular injury and transsphenoidal surgery. Front Horm Res. 2006;34:256-278.

16. Renn WH, Rhoton AL Jr. Microsurgical anatomy of the sellar region. J Neurosurg. 1975;43(3):288-298.

17. Fujii K, Chambers SM, Rhoton AL Jr. Neurovascular relationships of the sphenoid sinus. A microsurgical study. J Neurosurg. 1979; 50(1):31-39.

18. Koitschev A, Baumann I, Remy CT, et al. Rational CT diagnosis before operations on the paranasal sinuses. Article in German. HNO. 2002;50(3):217-222.

19. Laws ER Jr. Vascular complications of transsphenoidal surgery. Pituitary. 1999;2(2):163-170.

20. Cobb MI, Nimjee S, Gonzalez LF, et al. Direct repair of iatrogenic internal carotid artery injury during endoscopic endonasal approach surgery with temporary endovascular balloon-assisted occlusion: Technical case report. Neurosurgery. 2015;11(3)(suppl 3): E483-E487.

21. Al-Sheibani S, Zanation AM, Carrau RL, et al. Endoscopic endonasal transpterygoid nasopharyngectomy. Laryngoscope. 2011; 121(10):2081-2089

22. Kassam AB, Prevedello DM, Carrau RL, et al. Endoscopic endonasal skull base surgery: analysis of complications in the authors' initial 800 patients. J Neurosurg. 2011;114(6): 1544-1568

23. Padhye V, Valentine R, Paramasivan S, et al. Early and late complications of endoscopic hemostatic techniques following different carotid artery injury characteristics. Int Forum Allergy Rhinol. 2014:4(8):651-657.

24. Di Somma A, de Notaris M, Enseñat J, et al. The ventral route to intracranial aneurysm: from the origin towards modern transsphenoidal surgery. An historical review and current perspective. Rhinology. 2014;52(3):195-207.

25. Xiao LM, Tang B, Xie SH, et al. Endoscopic endonasal clipping of anterior circulation aneurysm: surgical techniques and results. World Neurosurg. 2018;115:e33-e44. 
26. Wang W-H, Lieber S, Lan M-Y, et al. Nasopharyngeal muscle patch for the management of internal carotid artery injury in endoscopic endonasal surgery. J Neurosurg. 2020;133(5): 1382-1387.

27. Shen J, Hur K, Zhang Z, et al. Objective validation of perfusion-based human cadaveric simulation training model for management of internal carotid artery injury in endoscopic endonasal sinus and skull base surgery. Oper Neurosurg (Hagerstown). 2018;15(2):231-238.

28. Duek I, Sviri GE, Amit M, et al. Endoscopic endonasal repair of internal carotid artery injury during endoscopic endonasal surgery. J Neurol Surg Rep. 2017;78(4):e125-e128.

29. Lum SG, Gendeh BS, Husain S, et al. Internal carotid artery injury during endonasal sinus surgery: our experience and review of the literature. Acta Otorhinolaryngol Ital. 2019;39(2):130-136.

30. Zhang HK, Ma N, Sun XC, et al. Endoscopic repair of the injured internal carotid artery utilizing oxidized regenerated cellulose and a free fascia lata graft. J Craniofac Surg. 2016;27(4):1021-1024.

31. Maza G, VanKoevering KK, Yanez-Siller JC, et al. Surgical simulation of a catastrophic internal carotid artery injury: a lasersintered model. Int Forum Allergy Rhinol. 2019;9(1):53-59.

32. Muto J, Carrau RL, Oyama K, et al. Training model for control of an internal carotid artery injury during transsphenoidal surgery. Laryngoscope. 2017;127(1):38-43.

33. Valentine R, Boase S, Jervis-Bardy J, et al. The efficacy of hemostatic techniques in the sheep model of carotid artery injury. Int Forum Allergy Rhinol. 2011;1(2):118-122.

34. Sylvester PT, Moran CJ, Derdeyn CP, et al. Endovascular management of internal carotid artery injuries secondary to endonasal surgery: case series and review of the literature. J Neurosurg. 2016;125(5):1256-1276.

35. Çinar C, Bozkaya H, Parildar M, et al. Endovascular management of vascular injury during transsphenoidal surgery. Interv Neuroradiol. 2013;19(1):102-109.

36. Zhang Y, Tian Z, Li C, et al. A modified endovascular treatment protocol for iatrogenic internal carotid artery injuries following endoscopic endonasal surgery. J Neurosurg. 2019;132(2): 343-350.

37. Willinsky RA, Taylor SM, TerBrugge K, et al. Neurologic complications of cerebral angiography: prospective analysis of 2,899 procedures and review of the literature. Radiology. 2003;227(2): 522-528.

38. Lim J, Suh SH, Lee K-Y, et al. Endovascular treatment of iatrogenic intracranial pseudoaneurysm following stent angioplasty. J Neuroimaging. 2012;22(2):194-196.
39. Griauzde J, Ravindra VM, Chaudhary N, et al. Use of the Pipeline embolization device in the treatment of iatrogenic intracranial vascular injuries: a bi-institutional experience. Neurosurg Focus. 2017;42(6):E9

40. Romero ADCB, Lal Gangadharan J, Bander ED, et al. Managing arterial injury in endoscopic skull base surgery: case series and review of the literature. Oper Neurosurg (Hagerstown). 2017; 13(1):138-149.

\section{Disclosures}

The authors report no conflict of interest concerning the materials or methods used in this study or the findings specified in this paper.

\section{Author Contributions}

Conception and design: Fustero de Miguel, López López, Orduna Martínez, Casado Pellejero. Acquisition of data: Fustero de Miguel, Avedillo Ruidiaz. Analysis and interpretation of data: Fustero de Miguel, López López, Avedillo Ruidiaz, Orduna Martínez. Drafting the article: Fustero de Miguel, López López, Avedillo Ruidíaz, Orduna Martínez. Critically revising the article: all authors. Reviewed submitted version of manuscript: Fustero de Miguel, Avedillo Ruidiaz, Orduna Martínez. Approved the final version of the manuscript on behalf of all authors: Fustero de Miguel. Administrative/technical/material support: Fustero de Miguel, Casado Pellejero, Moles Herbera. Study supervision: Fustero de Miguel, Moles Herbera.

\section{Supplemental Information \\ Videos}

Video 1. https://vimeo.com/481723883.

Previous Presentations

Presented as a poster at the XXI Congress of Spanish Society of Neurological Surgeons (Joint Meeting CNS, SBNS) held in Barcelona, Spain, in May 2017.

\section{Correspondence}

David Fustero de Miguel: University Hospital Miguel Servet, Zaragoza, Spain. fustero@hotmail.com. 